\title{
OXFORD
}

\section{Molecular Biology and Evolution}

\section{Adaptive Functional Diversification of Lysozyme in Insectivorous Bats}

\begin{tabular}{|r|l|}
\hline Journal: & Molecular Biology and Evolution \\
\hline Manuscript ID: & MBE-14-0286.R1 \\
\hline Manuscript Type: & Article \\
\hline Date Submitted by the Author: & 18 -Jul-2014 \\
\hline Complete List of Authors: & $\begin{array}{l}\text { Liu, Yang; East China Normal University, Institute of Molecular Ecology and } \\
\text { Evolution } \\
\text { He, Guimei; East China Normal University, Institute of Molecular Ecology } \\
\text { and Evolution } \\
\text { Xu, Huihui; East China Normal University, Institute of Molecular Ecology } \\
\text { and Evolution } \\
\text { Han, Xiuqun; East China Normal University, Institute of Molecular Ecology } \\
\text { and Evolution } \\
\text { Jones, Gareth; University of Bristol, School of Biological Sciences } \\
\text { Rossiter, Stephen; Queen Mary, } \\
\text { Zhang, Shuyi; East China Normal University, Institute of Molecular Ecology } \\
\text { and Evolution }\end{array}$ \\
\hline Key Words: & \begin{tabular}{l} 
Chiroptera, Iysozyme, diet, gene duplication, adaptive evolution \\
\hline
\end{tabular} \\
\hline
\end{tabular}




\section{Article: Discoveries}

\section{Adaptive Functional Diversification of Lysozyme in Insectivorous Bats}

Yang Liu, ${ }^{\dagger, 1}$ Guimei He, ${ }^{\dagger, 1}$ Huihui Xu, ${ }^{1}$ Xiuqun Han, ${ }^{1}$ Gareth Jones, ${ }^{2}$ Stephen J. Rossiter, ${ }^{*}, 3$ and Shuyi Zhang ${ }^{*}, 1$

1 Institute of Molecular Ecology and Evolution, Institutes for Advanced Interdisciplinary Research, East China Normal University, Shanghai 200062, China

${ }^{2}$ School of Biological Sciences, University of Bristol, Bristol BS8 1UG, United Kingdom

${ }^{3}$ School of Biological and Chemical Sciences, Queen Mary, University of London, London E1 4NS, United Kingdom

${ }^{\dagger}$ These authors contributed equally to this work

* To whom correspondence should be addressed. E-mails: s.j.rossiter@qmul.ac.uk or syzhang@bio.ecnu.edu.cn

Running title: Molecular Adaptation of Bat Lysozymes 


\begin{abstract}
The role of gene duplication in generating new genes and novel functions is well recognized and is exemplified by the digestion-related protein lysozyme. In ruminants, duplicated chicken-type lysozymes facilitate the degradation of symbiotic bacteria in the foregut. Chicken-type lysozyme has also been reported to show chitinase-like activity, yet no study has examined the molecular evolution of lysozymes in species that specialize on eating insects. Insectivorous bats number over 900 species, and lysozyme expression in the mouths of some of these species is associated with the ingestion of insect cuticle, suggesting a chitinase role. Here we show that chickentype lysozyme has undergone multiple duplication events in a major family of insecteating bats (Vespertilionidae) and that new duplicates have undergone molecular adaptation. Examination of duplicates from two insectivorous bats - Pipistrellus abramus and Scotophilus kuhlii - indicated that the new copy was highly expressed in the tongue, whereas the other one was less tissue-specific. Functional assays applied to pipistrelle lysozymes confirmed that, of the two copies, the tongue duplicate was more efficient at breaking down glycol chitin, a chitin derivative. These results suggest that the evolution of lysozymes in vespertilionid bats has likely been driven in part by natural selection for insectivory.
\end{abstract}

Key words: Chiroptera, lysozyme, diet, gene duplication, adaptive evolution. 


\section{Introduction}

Gene duplication is a widespread evolutionary mechanism that can lead to the generation of new genetic material as well as new biological functions (Ohno 1970; Zhang 2003; Innan and Kondrashov 2010). Following duplication, new genes may be lost, functionally conserved, or, in some cases, may undergo functional modifications or innovations (Zhang 2003). The enzyme lysozyme is considered a classic example of adaptive gene duplication, with duplicates detected in a wide range of animals (Cámara and Prieur 1984; Hammer, et al. 1987; Irwin and Wilson 1989; Kornegay 1996; Regel, et al. 1998).

The chicken-type lysozyme - which has been particularly well-studied - appears to function primarily in breaking down the peptidoglycan of bacterial cell walls, and is thus considered part of the immune response (Callewaert and Michiels 2010). In some taxa such as artiodactyl ruminants, the lysozyme also plays a role in facilitating digestion (Callewaert and Michiels 2010). The guts of these mammals play host to mutualistic symbiotic bacteria, which synthesize cellulases and thus allow nutrients to be obtained from otherwise indigestible plant material. In turn, the host expresses lysozymes to break down the dead bacteria and release further nutrients (Dobson, et al. 1984). As an adaptation for this so-called foregut fermentation, the cow appears to have evolved ten lysozyme duplicates, four of which are expressed in stomach (Irwin and Wilson 1989) where they are well-adapted to the highly acidic environment (Dobson, et al. 1984). Molecular evolutionary analyses show that these stomachspecific copies of ruminant lysozymes have been subject to concerted evolution, a process that probably acts to maintain and reinforce this specialized function (Irwin and Wilson 1990; Irwin, et al. 1993; Wen and Irwin 1999). Such patterns strongly contrast with those seen in the related yet omnivorous pig, which possesses just a single copy of the lysozyme gene (Yu and Irwin 1996).

Chicken-type lysozyme has been shown to have chitinase activity (Berger and Weiser 1957; Amano and Ito 1978; Li, et al. 2013) and, therefore, it is possible that apart from foregut-fermenters, adaptive gene duplication might also have occurred in species that ingest large amounts of chitin. Bats (Order Chiroptera) number over 1100 species and, while showing unparalleled dietary diversification among mammals, over 900 extant bat species are exclusively insectivorous (Simmons 2005), which is also considered the ancestral state of bats (Gunnell and Simmons 2005). Insectivorous bats appear to most commonly prey on insects from the orders Lepidoptera (moths and butterflies), Coleoptera (beetles) and Diptera (flies) (Hill and Smith 1984), and may consume around three quarters of their body mass per night (Kunz, et al. 1995) sometimes feeding on-the-wing. Given that bats must process their insect prey quickly, and that some prey possess chitinous exoskeletons that are hard to be chew (Evans and Sanson 2005), it follows that any biochemical breakdown of chitin to access its carbohydrate content could be highly adaptive. Supporting this idea, lysozyme expression in bat salivary glands correlates well with the extent of cuticle intake, suggesting that it may have been recruited for its chitinase activity in these taxa 
(Phillips, et al. 1998). Furthermore, chitinase has been detected in the intestines of vespertilionid bats (Whitaker, et al. 2004) and has also been shown to be active in the stomachs of several European bat species from the same family (Strobel, et al. 2013).

Here we present the first in-depth study of the molecular evolution of chicken-type lysozymes in bats, combining sequencing with analyses of gene expression profiles and enzyme function assays. We test the following three hypotheses. (i) chicken-type lysozyme will occur as multiple copies in insect-eating bats due to duplication events; (ii) new duplicate gene copies of lysozyme will show evidence of Darwinian selection consistent with molecular adaptation for new roles in processing chitin, and (iii) these new copies will show greater activity for breaking down chitin. 


\section{Results}

\section{Lysozyme Gene Duplications in Bats}

We sequenced the complete coding region of chicken-type lysozyme genes from 19 bat species. The coding part of bat lysozyme genes spanned $447 \mathrm{bp}$, except for two copies detected in the genus Myotis (MricLyz-4 and MadvLyz-4), both of which shared a 3 bp deletion. Available lysozyme gene sequences of two bat species, Myotis lucifugus $(\mathrm{n}=3)$ and Pteropus vampyrus $(\mathrm{n}=1)$, were retrieved from the Ensembl genome database (www.ensembl.org). In total, taxonomic coverage included nine bat families, comprising eight families of insectivorous bats as well as the Pteropodidae (Old World fruit bats). In seven of the families surveyed, cloning yielded a single lysozyme gene copy. In contrast, multiple duplicated genes were found in the insectivorous family Vespertilionidae, with potential duplications detected in the genera Ia, Myotis, Murina, Nyctalus, Pipistrellus, Scotophilus and Scotomanes. Additionally, one duplication event was also uncovered in the pteropodid Cynopterus sphinx (supplementary table S1).

\section{Molecular Evolution of Bat Lysozyme Genes}

Gene trees generated by Bayesian, maximum likelihood (ML), neighbor-joining (NJ) and maximum parsimony (MP) methods revealed several independent duplication events in bats (fig. 1 and supplementary fig. S1). Topological differences were observed among gene trees produced by the different tree-building methods. Overall the gene copies clustered together in their respective sub-ordinal groups with the exception of the two yangochiropterans Taphozous melanopogon and Tadarida plicata. Both of these species were correctly recovered in the Yangochiroptera in the NJ tree (supplementary fig. S1b), but either one or both of them were in the Yinpterochiroptera in the Bayesian, ML and MP trees (with low statistical support), suggesting inconsistencies arise from methodological artefacts (fig. 1 and supplementary fig. S1).

Within the Yangochiroptera, all genes from vespertilionid bats grouped in a wellsupported clade across all methods, and, within the genus Myotis, we detected at least three gene duplications that have given rise to four main sub-groups, which we refer to as $L y z-1, L y z-2, L y z-3$ and $L y z-4$. Further duplication events were detected in Pipistrellus and Nyctalus, occurring before the split of the two genera, and in the clade containing Ia io and Scotomanes ornatus. Independent gene duplications were also observed in either Scotophilus kuhlii or Murina leucogaster (fig. 1 and supplementary fig. S1). No gene conversion was detected among paralogs from one species.

We tested whether the duplicated genes in bats were associated with molecular adaptation, which would be consistent with the recruitment of derived genes to perform new roles, such as in digestion. By estimating the ratio of the rate of nonsynonymous substitutions to the rate of synonymous ones (termed omega, $\omega$ ) we found several episodes of positive selection in the putative new bat genes, mainly in the family Vespertilionidae. In the majority of these cases we observed that one gene 
copy (e.g. $L y z-1$ in this study) was found to be under purifying selection $(\omega<1)$ with few nucleotide substitutions, whereas the other gene(s) (e.g. Lyz-2, Lyz-3 or Lyz-4) had undergone positive selection $(\omega>1)$. Overall our selection tests showed functional conservation of one gene copy and adaptation in the other duplicate(s) (supplementary fig. S2 and supplementary table S2).

\section{Expression Profiles of Duplicated Genes}

We characterized lysozyme expression patterns for two insectivorous bats, Pipistrellus abramus and Scotophilus kuhlii (Vespertilionidae), and one frugivorous bat, Cynopterus sphinx (Pteropodidae). The Lyz-1 gene was expressed in most organs examined in all three species, whereas $L y z-2$ showed more tissue-specific expression profiles. In $P$. abramus, the PabrLyz-2 gene expression was detected in the spleen, stomach, intestine and tongue, with the peak expression value in the tongue, in contrast to PabrLyz-1 in which there was no detected expression in the tongue (fig. 2a). In S. kuhlii, SkuhLyz-2 also showed highest expression in the tongue, although this gene was also more widely expressed in other organs compared to PabrLyz-2 (fig. 2b). In C. sphinx, the CsphLyz-2 gene showed extremely low expression level $(0.0075 \pm 0.0073)$ in the tongue and was not detected in any other organ types examined (fig. 2c).

\section{Enzymatic Activities of Pipistrelle Lysozymes}

Recombinant lysozymes based on PabrLyz-1 and PabrLyz-2 genes were synthesized individually and their enzymatic activities were measured. To determine the assumed original anti-bacterial function of the gene, we used Micrococcus lysodeikticus as a substrate. We found that the enzymes differed in their enzymatic ability to breakdown bacteria (one-way ANOVA, $P=0.015$ ), with PabrLyz-2 lysozyme showing significantly lower activity than hen egg-white lysozyme (HEWL) (Holm-Sidak, $P=$ 0.017). No significant differences were found between PabrLyz-1 and PabrLyz-2 (Holm-Sidak, $P=0.507$ ) or between PabrLyz-1 HEWL (Holm-Sidak, $P=0.054$ ) (fig. 3a).

To test whether bat lysozymes, especially the PabrLyz-2, showed a chitinolytic activity consistent with a hypothesized chitinase role, we quantified the performance of the protein at breaking down the substrate glycol chitin. The results again revealed significant overall differences in hydrolytic activity (one-way ANOVA, $P<0.001$ ). PabrLyz-2 exhibited significantly higher lytic efficiency than PabrLyz-1 (Holm-Sidak, $P<0.001$ ), the efficiency of PabrLyz-1 was significantly higher than chitinase (HolmSidak, $P=0.005)$, and the efficiency of PabrLyz-2 was significantly higher than chitinase (Holm-Sidak, $P<0.001$ ) (fig. 3b). Details of one-way ANOVAs are listed in supplementary table S3. 


\section{Discussion}

We undertook the first survey of lysozyme genes in bats and found evidence of widespread duplications having occurred in a major clade of insectivorous species (Vespertilionidae). In this group, which represents the most speciose family of bats (Simmons 2005), nine of the ten species examined possessed two or more chickentype lysozyme copies, thus supporting our initial hypothesis. The only exception was Barbastella beijingensis, which appeared to have only one gene copy. In contrast, bats from all other families examined, from both the Yinpterochiroptera and Yangochiroptera suborders (Teeling, et al. 2005), possessed just a single lysozyme gene, again with one exception: two copies were detected in the Old World fruit bat Cynopterus sphinx.

In phylogenetic reconstructions based on bat lysozyme sequences, duplicated genes in bats did not always group together within taxa, in contrast to the situation seen in ruminants, where coding regions of lysozyme genes from the same species have been subject to concerted evolution (Irwin and Wilson 1990). In vespertilionid bats, lysozyme gene trees showed conflicts with known species relationships; to reconcile this discordance we conclude that independent gene duplications predated the split between the genera Pipistrellus and Nyctalus and also between the genera Scotomanes and $I a$. Further gene duplications also occurred independently in the genera Scotophilus, Murina and Myotis. Due to the low support for several nodes in the gene trees, it is not possible to estimate the exact number of gene duplications in this family. At the same time, however, we cannot completely rule out the potential for some differential representation of particular copies due to biases in the gene amplification and cloning process. Previous studies have shown that duplicated lysozymes perform a digestive function in the stomachs of foregut fermenting animals (Irwin and Wilson 1989; Kornegay 1996), and that gene conversion has acted to constrain this role in ruminants (Irwin and Wilson 1990). Despite this we found no evidence of gene conversion in species with multiple lysozyme genes.

The finding that branches leading to duplicated gene copies in the vespertilionid clade (Lyz-2, $L y z-3$, or $L y z-4$ genes) were characterized by positive selection, whereas those leading to the $L y z-1$ genes were under purifying selection, supports our second hypothesis that the observed lysozyme duplicates in bats are adaptive. We suggest that conserved sequence variation in $L y z-1$ genes could point to a function in host defense (Callewaert and Michiels 2010). On the other hand, the other derived gene copies in insectivorous bats have likely been recruited to perform new biological functions probably in digestion. Several studies have reported new digestive functions of lysozymes in foregut-fermenting animals (Dobson, et al. 1984; Beintema 1990; Kornegay, et al. 1994). Similar functional shifts are also found in some invertebrate species, including goose- and invertebrate-types of lysozyme too (Regel, et al. 1998; Xue, et al. 2010; Wang, et al. 2012), although no such evidence has yet been reported in bats. 
Expression patterns of lysozyme genes support the expectation that duplicated lysozymes in bats are possibly related to chitin digestion activity (Phillips, et al. 1998). Although based on small sample sizes, we found that the $L y z-2$ genes from both Pipistrellus and Scotophilus showed exceptionally high expression in the tongue, compared with less tissue-specific expression patterns in the Lyz-1 copies that are more consistent with a general function in immunity. Ideally larger sample sizes are desirable to verify these results, and also to determine whether expression occurs throughout the tongue or whether it is concentrated in the exocrine von Ebner's gland (Reitamo, et al. 1977). While we recorded low Lyz-2 expression levels in the stomach (fig. 2a and b), functional assays revealed the duplicate copy continued to be active at low $\mathrm{pH}$ (fig. $3 \mathrm{~b}$ ). Consequently it is possible that Lyz-2 from the tongue continues to function after being swallowed, where its role would be augmented by stomach chitinase (Strobel, et al. 2013). Our results from experimental assays also add weight to a role of Lyz-2 in digestion; in particular, the Lyz-2 in P. abramus displayed significantly greater lytic efficiency for glycol chitin than did PabrLyz-1, whereas anti-bacterial activity in PabrLyz-2 was similar to that of PabrLyz-1. A degree of functional redundancy in the derived copy, which has been reported in RNase1 (Zhang, et al. 2002), could provide more favorable conditions for natural selection to promote a modified role.

Given our results from insect-eating bats, it is noteworthy that we observed markedly different expression patterns for the two duplicated lysozyme genes observed within the frugivorous bat Cynopterus sphinx. Specifically, the CsphLyz-1 gene was seen to be expressed widely across different tissue types, whereas the CsphLyz-2 showed very weak expression in the tongue. The low expression level of CsphLyz-2 could point to non-functionality, although we cannot rule out the possibility it is expressed elsewhere. If the former case is correct then high $\omega$ value (2.67) in the fruit bat lineage could be the consequence of relaxed selective constraint (supplementary table S2). Although $C$. sphinx, like the flying fox Pteropus giganteus, may host symbiotic bacteria to assist with the digestion of cellulose and xylan in leaves (Ruby, et al. 2000; Prem Anand and Sripathi 2004), we found no evidence that the duplicated lysozyme in C. sphinx plays a similar function to that found in the foreguts of some ungulates and primates (Dobson, et al. 1984; Beintema 1990).

Chitin is a major component of insect cuticle (Andersen 1979) and poses challenges to insect-eating species such as bats that must consume large numbers of insects each night (Kunz 1974; Anthony and Kunz 1977; Encarnacao and Dietz 2006). The cuticles of some insects such as beetles present particular difficulties to process and digest (Evans and Sanson 2005). Some bats are known to remove chitinous parts of insects such as wings and legs before eating the softer body parts (Barclay, et al. 1991), while other species are thought to breakdown chitin using chitinases, which may be either synthesized by symbiotic bacteria in the intestine (Whitaker, et al. 2004) or expressed endogenously in the stomach (Strobel, et al. 2013). Although the harder parts of insect exoskeletons might not necessarily be the most chitin-rich (Richards 1951), Phillips et 
al. (1998) found a relationship between lysozyme-reactivity in bat salivary glands and feeding on hard-bodied insects.

Our results from vespertiolinid bats provide the best evidence to date for a chitinaselike function of some lysozymes in bats. Beetles are frequently eaten by Pipistrellus, Nyctalus, Murina, and Ia species (Jones 1995; Lee and Lee 2005; Thabah, et al. 2007; Ma, et al. 2008), whereas Barbastella - in which gene duplication was absent - is known to specialize on softer bodied insects such as moths (Rydell, et al. 1996). Apart from fish, beetles are also the main prey of Myotis ricketti (Ma, et al. 2006), although the congeners $M$. siligorensis and $M$. lucifugus are thought not to specialize on beetles (Wei, et al. 2006; Burles, et al. 2008). Our integrated results suggest that gene duplication with subsequent functional modification of lysozymes in vespertilionid bats is likely to have contributed to the success and diversification of insect-eating bats. At the same time however, a chitinolytic function for lysozyme might not solely be an adaptation for diet. Indeed it is noteworthy that chitin is also a major component of the cell walls of some fungi (Bartnicki-Garcia 1968). Because some fungi species, including Geomyces destructans that causes white-nose syndrome (Blehert, et al. 2009), represent potential threats to bats, we cannot rule out the possibility that duplications of lysozyme genes have in part been driven for immune function. 


\section{Materials and Methods \\ Molecular Cloning and Gene Sequencing}

To study the molecular evolution of bat lysozymes we undertook polymerase chain reactions (PCR) and cloned the amplified products for 19 species of bat. Using RNAiso reagent (TaKaRa), we isolated total RNA from the following archived bat organs in $-80^{\circ} \mathrm{C}$ refrigerator: liver (from 18 species), intestine (18 species), tongue (16 species), kidney (3 species), stomach (3 species), spleen (2 species) and lung (2 species). First-strand cDNA was synthesized with reverse transcriptase using the SuperScript III kit (Invitrogen). PCR primers used were Lyz-F (5' GGT CTG GCT TCG CAG TCA AC 3') and Lyz-R (5' GAG AGA AAA GGA GCT GAA GAA GG 3'). The PCR protocol was: $5 \mathrm{~min}$ at $95^{\circ} \mathrm{C}$, then 30 cycles of $30 \mathrm{~s}$ at $95^{\circ} \mathrm{C}, 30 \mathrm{~s}$ at $54^{\circ} \mathrm{C}$ and $40 \mathrm{~s}$ at $72^{\circ} \mathrm{C}$, and with a $10 \mathrm{~min}$ extension at $72^{\circ} \mathrm{C}$. For cloning we used the pMD19-T vector (TaKaRa) and all clones were sequenced on an ABI 3730 (Applied Biosystems). Repeated PCRs were carried out in each species to screen and verify the duplicated genes cloned. In addition, lysozyme sequences from $P$. vampyrus and $M$. lucifugus were obtained by BLAT searches of the Ensembl database (www.ensembl.org). Detailed species and gene information is given in supplementary table S1.

\section{Phylogenetic Reconstruction and Selection Test}

For phylogenetic reconstruction, the $\mathrm{TVM}+\Gamma$ nucleotide substitution model was selected based on Akaike information criterion in ModelTest Server (Posada 2006). We undertook Bayesian phylogenetic inference using MrBayes 3.1.2 software (Ronquist and Huelsenbeck 2003), ML inference using RAxML 7.0.4 (Stamatakis 2006), NJ and MP inferences using MEGA 5 (Tamura, et al. 2011). For Bayesian inference, five million MCMC generations were performed with the first two million discarded as burn-in. For ML, 200 topologies were searched and 1000 bootstrap replications performed, and for NJ and MP, 2000 bootstrap replicates were performed. We tested for evidence of gene conversion using the software GENECONV (Sawyer 1989).

To test for positive selection on bat lineages, we estimated the ratio of the rate of nonsynonymous substitutions to the rate of synonymous substitutions $(\omega)$. Values of $\omega$ that exceed 1 indicate positive selection. We implemented the free-ratio model in PAML version 4 in which $\omega$ is estimated independently for each branch in the tree (Yang 2007). A likelihood ratio test was used to compare the fit of the free-ratio model to that of one-ratio model in which $\omega$ was the same across all branches (Yang 1998). The gene tree from figure 1 was used as the given topology in selection analyses.

\section{Quantitative Real-Time PCR}

To determine the expression profiles of the duplicated genes, we performed quantitative real-time PCR (qPCR) experiments on an ABI 7300 (Applied Biosystems) for two insectivorous bats (P. abramus and S. kuhlii) and one fruit bat (C. sphinx). 
Two adult and non-pregnant individuals per species were captured in the field. A total of nine tissue types (heart, liver, spleen, lung, kidney, muscle, intestine, stomach and tongue) were sampled and immediately flash frozen in liquid nitrogen before being transferred to $-80^{\circ} \mathrm{C}$ refrigerator in lab. The $\mathrm{qPCR}$ primers and probes were designed using the software Primer Express (Applied Biosystems) and the detailed information is given in supplementary table S4. For qPCRs, the glyceraldehyde-3-phosphate dehydrogenase gene $(G A P D H)$ was used as a control. The $5^{\prime}$-ends of the TaqMan probes were labeled with reporter dye (6-carboxyfluorescein; FAM) and the 3'-end with quenching dye (6-carboxytetramethylrhodamine; TAMRA). Note the 3 ' ends of the two Scotophillus probes were labeled with a minor groove binder (MGB) and a non-fluorescent quencher rather than TAMRA.

Efficiencies of lysozyme and $G A P D H$ genes were determined by qPCR using a serial dilution of cDNA samples (Livak and Schmittgen 2001). The amplification efficiencies of the two genes were close to $100 \%$ and thus relative gene expression analysis could be applied using qPCR. The PCR was carried out in $20 \mu$ l volume containing $10 \mu 1$ TaqMan master mix, $0.4 \mu 1$ Rox Reference Dye, 900nM primers, 100200nM TaqMan probe, and $1 \mu \mathrm{l}$ cDNA sample. The PCR protocol was: $20 \mathrm{~s}$ at $95^{\circ} \mathrm{C}$, then 40 cycles of $8 \mathrm{~s}$ at $95^{\circ} \mathrm{C}$ and $31 \mathrm{~s}$ at $60.5^{\circ} \mathrm{C}$. Each cDNA sample was amplified by three independent PCRs. All data were analyzed using Sequence Detector Systems software (Applied Biosystems). The average threshold cycle $(\Delta \mathrm{Ct})$ values of $L y z-1$ genes in muscle were used for calibration. To compare tissue-specific gene expression levels, we used the $2^{-\Delta \Delta \mathrm{Ct}}$ method in which all $\Delta \mathrm{Ct}$ values were compared to the value obtained for muscle (Livak and Schmittgen 2001).

\section{Functional Assay on Recombinant Protein}

To determine the putative function of bat lysozyme, we expressed both the PabrLyz-1 and PabrLyz-2 genes from the insectivorous bat P. abramus, the latter of which showed high expression in the tongue. The two pipistrelle genes were ligated into the $\mathrm{NcoI}$ and $\mathrm{XhoI}$ sites of a pET-28a (+) vector (Novagen) respectively. The recombinant plasmids were then transformed into the $E$. coli strain Rosetta (DE3). Recombinant proteins were purified and quantified following published methods (Baneyx 1999; Supungul, et al. 2010).

We quantified the bacteriolytic activity of the recombinant proteins using a modified version of the protocol described in Kikuchi et al. (1988). Here we used $180 \mu$ l of $0.2 \mu \mathrm{g} / \mu 1 \mathrm{M}$. lysodeikticus (Sigma-Aldrich) in phosphate-citrate buffer with $\mathrm{pH}$ values ranging from 4.2 to 8.0 ( $0.2 \mathrm{M}$ disodium hydrogen phosphate and $0.1 \mathrm{M}$ citric acid). Then $4 \mu \mathrm{g}$ lysozyme was mixed with the solution quickly to a final volume of $200 \mu \mathrm{l}$. The initial absorbance value $\left(\mathrm{A}_{0}\right)$ at $450 \mathrm{~nm}$ was measured immediately using an iMark microplate reader (Bio-Rad). The solution was then incubated at $37^{\circ} \mathrm{C}$ for $30 \mathrm{~min}$ with a final ice bath to stop the reaction. The absorbance value $\left(\mathrm{A}_{1}\right)$ was measured again immediately after reaction termination. One unit of lysozyme activity (U) is defined as the amount of enzyme causing a 0.001 decrease of absorbance value 
at 450nm per minute (Kikuchi, et al. 1988). HEWL (Sigma-Aldrich) was used as a positive control and the wells contained all the reagents except the enzyme was used as a blank control.

To assess the chitinolytic activity of the bat lysozymes, we undertook assays using the chitin-derived substrate glycol chitin ( $\sim 90 \%$ degree of acetylation, Polyscitech), which is commonly used as a substitute for non-soluble chitin. In the assay we added $4 \mu \mathrm{g}$ of purified lysozyme to $100 \mu \mathrm{l}$ of $0.05 \%$ substrate with $0.1 \mathrm{M}$ acetic acid-sodium acetate buffer ( $\mathrm{pH}$ values at 5.0, 6.0, 7.0 and 8.0 respectively). The solution was incubated for $30 \mathrm{~min}$ at $37^{\circ} \mathrm{C} .200 \mu \mathrm{l}$ of color reagent $(0.5 \mathrm{~g}$ of potassium ferricyanide dissolved in $1 \mathrm{~L}$ of $0.5 \mathrm{M}$ sodium carbonate) was added and boiled for $15 \mathrm{~min}$. To determine the activity of chitinolytic hydrolysis we examined the absorbance at $420 \mathrm{~nm}\left(\mathrm{~A}_{1}\right)$, which reflects the quantity of the product $\mathrm{N}$-acetylglucosamine (Imoto and Yagishita 1971). We included a positive control that contained chitinase (SigmaAldrich) and a negative control that contained no enzyme but all other reagents $\left(\mathrm{A}_{0}\right)$. One $U$ is defined as the amount of enzyme that causes a decrease in absorbance of 0.001 at $420 \mathrm{~nm}$ per minute. 


\section{Figure Legends}

Fig. 1. Bayesian tree based on lysozyme sequences of bats. Node support values are Bayesian posterior probabilities. Colored dots indicate inferred major duplication events in bats. The duplicated genes MsilLyz-3, MleuLyz-3 and CsphLyz-2 are marked by colored diamonds, which show ambiguous positions in the tree. Genes from the family Vespertilionidae are highlighted within a box. Sequences from species of the divergent suborder Yinpterochiroptera were used to root the tree (black arrow), however, Taphozous melanopogon is incorrectly grouped in the suborder. Species used for subsequent expression and functional assays are also indicated. Full species names and gene symbols are given in supplementary table S1.

Fig. 2. Gene expression pattern in Pipistrellus abramus (a), Scotophilus kuhlii (b) and Cynopterus sphinx (c), respectively. In each plot, the two duplicates $L y z-1$ and $L y z-2$ are represented by black and grey bars, respectively. For the latter duplicate CsphLyz2, expression was too low to show on the graph. Error bars indicate standard deviations. The organ symbols are muscle (MU), stomach (ST), intestine (IN), spleen (SP), lung (LU), kidney (KI), liver (LI), tongue (TO) and heart (HE). Symbol “*” represent expression fold changes were obtained by comparisons with the value for muscle.

Fig. 3. Enzymatic activities of pipistrelle lysozymes. For the two recombinant lysozymes PabrLyz-1 and PabrLyz-2 (represented by light and broken dark blue, respectively), potential anti-bacterial activity was assayed using the substrate $M$. lysodeikticus, and chitinase activity was assayed using glycol chitin. Positive controls (grey line) were HEWL for M. lysodeikticus and chitinase for glycol chitin. Error bars indicate standard deviations. 


\section{Supplementary Material}

Supplementary figures S1 and S2 and tables S1-S4 are available at Molecular Biology and Evolution online (http://www.mbe.oxfordjournals.org/).

\section{Acknowledgements}

We thank Yi-Hsuan Pan for comments on the lysozyme protein assays, James A. Cotton for valuable suggestions at the early stage of this work, and Junpeng Zhang for bat sample collection during field work. This work was supported by the National Natural Science Foundation of China (31172077 to S.Z.), an East China Normal University Fostering Project for National Top Hundred Doctoral Dissertations (PY2011012 to Y.L.), an Innovation Fund of East China Normal University (78210200 to G.H.), and a Biotechnology and Biological Sciences Research Council China Partnering Award (BB/G530392/1 to G.J., S.J.R. and S.Z.). The GenBank accession numbers for new lysozyme genes sequenced in this paper are JX064159JX064193. 


\section{References}

Amano K, Ito E. 1978. The action of lysozyme on partially deacetylated chitin. Eur J Biochem 85:97104.

Andersen SO. 1979. Biochemistry of insect cuticle. Ann Rev Entomol 24:29-61.

Anthony ELP, Kunz TH. 1977. Feeding strategies of the little brown bat, Myotis lucifugus, in southern New Hampshire. Ecology 58:775-786.

Baneyx F. 1999. Recombinant protein expression in Escherichia coli. Curr Opin Biotechnol 10:411421.

Barclay RMR, Dolan M, Dyck A. 1991. The digestive efficiency of insectivorous bats. Can J Zool 69:1853-1856.

Bartnicki-Garcia S. 1968. Cell wall chemistry, morphogenesis, and taxonomy of fungi. Annu Rev Microbiol 22:87-108.

Beintema JJ. 1990. The primary structure of langur (Presbytis entellus) pancreatic ribonuclease: adaptive features in digestive enzymes in mammals. Mol Biol Evol 7:470-477.

Berger LR, Weiser RS. 1957. The beta-glucosaminidase activity of egg-white lysozyme. Biochim Biophys Acta 26:517-521.

Blehert DS, Hicks AC, Behr M, Meteyer CU, Berlowski-Zier BM, Buckles EL, Coleman JT, Darling SR, Gargas A, Niver R, et al. 2009. Bat white-nose syndrome: an emerging fungal pathogen? Science 323:227.

Burles DW, Brigham RM, Ring RA, Reimchen TE. 2008. Diet of two insectivorous bats, Myotis lucifugus and Myotis keenii, in relation to arthropod abundance in a temperate Pacific Northwest rainforest environment. Can J Zool 86:1367-1375.

Callewaert L, Michiels CW. 2010. Lysozyme in the animal kingdom. J Biosci 35:127-160.

Cámara VM, Prieur DJ. 1984. Secretion of colonic isozyme of lysozyme in association with cecotrophy of rabbits. Am J Physiol 247:G19-G23.

Dobson DE, Prager EM, Wilson AC. 1984. Stomach lysozymes of ruminants. I. Distribution and catalytic properties. J Biol Chem 259:11607-11616.

Encarnacao JA, Dietz M. 2006. Estimation of food intake and ingested energy in Daubenton's bats (Myotis daubentonii) during pregnancy and spermatogenesis. Eur J Wildl Res 52:221-227.

Evans AR, Sanson GD. 2005. Biomechanical properties of insects in relation to insectivory: cuticle thickness as an indicator of insect 'hardness' and 'intractability'. Aust J Zool 53:9-19.

Gunnell GF, Simmons NB. 2005. Fossil evidence and the origin of bats. J Mammal Evol 12:209-246.

Hammer MF, Schilling JW, Prager EM, Wilson AC. 1987. Recruitment of lysozyme as a major enzyme in the mouse gut: duplication, divergence, and regulatory evolution. J Mol Evol 24:272-279.

Hill JE, Smith JD. 1984. Bats a natural history. London: British Museum (Natural History).

Imoto T, Yagishita K. 1971. A simple activity measurement of lysozyme. Agr Biol Chem 35:1154-1156.

Innan H, Kondrashov F. 2010. The evolution of gene duplications: classifying and distinguishing between models. Nat Rev Genet 11:97-108.

Irwin DM, White RT, Wilson AC. 1993. Characterization of the cow stomach lysozyme genes: repetitive DNA and concerted evolution. J Mol Evol 37:355-366.

Irwin DM, Wilson AC. 1990. Concerted evolution of ruminant stomach lysozymes. Characterization of lysozyme cDNA clones from sheep and deer. J Biol Chem 265:4944-4952.

Irwin DM, Wilson AC. 1989. Multiple cDNA sequences and the evolution of bovine stomach lysozyme. J Biol Chem 264:11387-11393. 
Jones G. 1995. Flight performance, echolocation and foraging behaviour in noctule bats Nyctalus noctula. J Zool 237:303-312.

Kikuchi M, Yamamoto Y, Taniyama Y, Ishimaru K, Yoshikawa W, Kaisho Y, Ikehara M. 1988. Secretion in yeast of human lysozymes with different specific activities created by replacing valine-110 with proline by site-directed mutagenesis. Proc Natl Acad Sci USA 85:9411-9415.

Kornegay JR. 1996. Molecular genetics and evolution of stomach and nonstomach lysozymes in the hoatzin. J Mol Evol 42:676-684.

Kornegay JR, Schilling JW, Wilson AC. 1994. Molecular adaptation of a leaf-eating bird: stomach lysozyme of the hoatzin. Mol Biol Evol 11:921-928.

Kunz TH. 1974. Feeding ecology of a temperate insectivorous bat (Myotis velifer). Ecology 55:693-711.

Kunz TH, Whitaker JO, Wadanoli MD. 1995. Dietary energetics of the insectivorous Mexican freetailed bat (Tadarida brasiliensis) during pregnancy and lactation. Oecologia 101:407-415.

Lee Y, Lee L. 2005. Food habits of Japanese pipistrelles Pipistrellus abramus (Chiroptera: Vespertilionidae) in northern Taiwan. Zool Stud 44:95-101.

Li Z, Cho S, Kwon IC, Janat-Amsbury MM, Huh KM. 2013. Preparation and characterization of glycol chitin as a new thermogelling polymer for biomedical applications. Carbohydr Polym 92:2267-2275.

Livak KJ, Schmittgen TD. 2001. Analysis of relative gene expression data using real-time quantitative PCR and the $2^{-\triangle \Delta C T}$ method. Methods 25:402-408.

Ma J, Liang B, Zhang S, Metzner W. 2008. Dietary composition and echolocation call design of three sympatric insectivorous bat species from China. Ecol Res 23:113-119.

Ma J, Zhang J, Liang B, Zhang L, Zhang S, Metzner W. 2006. Dietary characteristics of Myotis ricketti in Beijing, north China. J Mammal 87:339-344.

Ohno S. 1970. Evolution by gene duplication. New York: Springer.

Phillips CJ, Weiss A, Tandler B. 1998. Plasticity and patterns of evolution in mammalian salivary glands: comparative immunohistochemistry of lysozyme in bats. Eur J Morphol 36:19-26.

Posada D. 2006. ModelTest Server: a web-based tool for the statistical selection of models of nucleotide substitution online. Nucleic Acids Res 34:W700-W703.

Prem Anand AA, Sripathi K. 2004. Digestion of cellulose and xylan by symbiotic bacteria in the intestine of the Indian flying fox (Pteropus giganteus). Comp Biochem Physiol A 139:65-69.

Regel R, Matioli SR, Terra WR. 1998. Molecular adaptation of Drosophila melanogaster lysozymes to a digestive function. Insect Biochem Mol Biol 28:309-319.

Reitamo S, Klockars M, Raeste AM. 1977. Immunohistochemical identification of lysozyme in minor salivary-glands of man. Arch Oral Biol 22:515-519.

Richards AG. 1951. The integument of arthropods. The chemical components and their properties. The anatomy and development. The permeability. Minneapolis: University of Minnesota Press.

Ronquist F, Huelsenbeck JP. 2003. MrBayes 3: Bayesian phylogenetic inference under mixed models. Bioinformatics 19:1572-1574.

Ruby J, Nathan PT, Balasingh J, Kunz TH. 2000. Chemical composition of fruits and leaves eaten by short-nosed fruit bat Cynopterus sphinx. J Chem Ecol 26:2825-2841.

Rydell J, Natuschke G, Theiler A, Zingg PE. 1996. Food habits of the barbastelle bat Barbastella barbastellus. Ecography 19:62-66.

Sawyer S. 1989. Statistical tests for detecting gene conversion. Mol Biol Evol 6:526-538.

Simmons NB. 2005. Order Chiroptera. In: Wilson DE, Reeder MD, editors. Mammal species of the 
World: A taxonomic and geographic reference. Baltimore: The Johns Hopkins University Press.

Stamatakis A. 2006. RAxML-VI-HPC: maximum likelihood-based phylogenetic analyses with thousands of taxa and mixed models. Bioinformatics 22:2688-2690.

Strobel S, Roswag A, Becker NI, Trenczek TE, Encarnacao JA. 2013. Insectivorous bats digest chitin in the stomach using acidic mammalian chitinase. PLoS One 8:e72770.

Supungul P, Rimphanitchayakit V, Aoki T, Hirono I, Tassanakajon A. 2010. Molecular characterization and expression analysis of a c-type and two novel muramidase-deficient i-type lysozymes from Penaeus monodon. Fish Shellfish Immunol 28:490-498.

Tamura K, Peterson D, Peterson N, Stecher G, Nei M, Kumar S. 2011. MEGA5: molecular evolutionary genetics analysis using maximum likelihood, evolutionary distance, and maximum parsimony methods. Mol Biol Evol 28:2731-2739.

Teeling EC, Springer MS, Madsen O, Bates P, O'Brien S J, Murphy WJ. 2005. A molecular phylogeny for bats illuminates biogeography and the fossil record. Science 307:580-584.

Thabah A, Li G, Wang Y, Liang B, Hu K, Zhang S, Jones G. 2007. Diet, echolocation calls, and phylogenetic affinities of the great evening bat (Ia io; Vespertilionidae): another carnivorous bat. J Mammal 88:728-735.

Wang Q, Zhang L, Zhao J, You L, Wu H. 2012. Two goose-type lysozymes in Mytilus galloprovincialis: possible function diversification and adaptive evolution. PLoS One 7:e45148.

Wei L, Zhou S, Zhang L, Liang B, Hong T, Zhang S. 2006. Characteristics of echolocation calls and summer diet of three sympatric insectivorous bats species. Zool Res 27:235-241.

Wen Y, Irwin DM. 1999. Mosaic evolution of ruminant stomach lysozyme genes. Mol Phylogenet Evol 13:474-482.

Whitaker JO, Dannelly HK, Prentice DA. 2004. Chitinase in insectivorous bats. J Mammal 85:15-18.

Xue Q, Hellberg ME, Schey KL, Itoh N, Eytan RI, Cooper RK, La Peyre JF. 2010. A new lysozyme from the eastern oyster, Crassostrea virginica, and a possible evolutionary pathway for i-type lysozymes in bivalves from host defense to digestion. BMC Evol Biol 10:213.

Yang Z. 1998. Likelihood ratio tests for detecting positive selection and application to primate lysozyme evolution. Mol Biol Evol 15:568-573.

Yang Z. 2007. PAML 4: phylogenetic analysis by maximum likelihood. Mol Biol Evol 24:1586-1591.

Yu M, Irwin DM. 1996. Evolution of stomach lysozyme: the pig lysozyme gene. Mol Phylogenet Evol 5:298-308.

Zhang J. 2003. Evolution by gene duplication: an update. Trends Ecol Evol 18:292-298.

Zhang J, Zhang YP, Rosenberg HF. 2002. Adaptive evolution of a duplicated pancreatic ribonuclease gene in a leaf-eating monkey. Nat Genet 30:411-415. 
Myotis

MsilLyz-3

Scotophilus

Ia and Scotomanes

Murina

MleuLyz-3

Nyctalus and

Pipistrellus

CsphLyz-2

real-time PCR

enzymatic assay
Molecular Biology and Evolution

Page 18 of 20

$1.00[$ MadvLyz-4

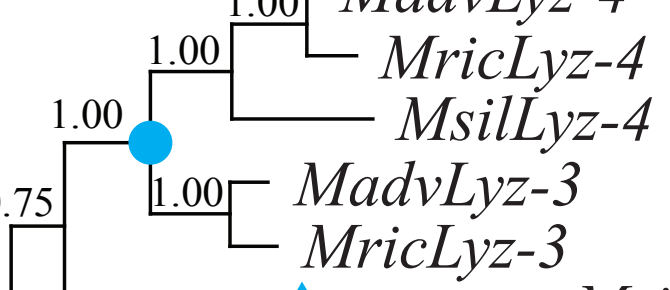

0.80 MsilLyz-3 $1.00-M a d v L y z-2$

$0.77 \longleftarrow$ MricLyz-2

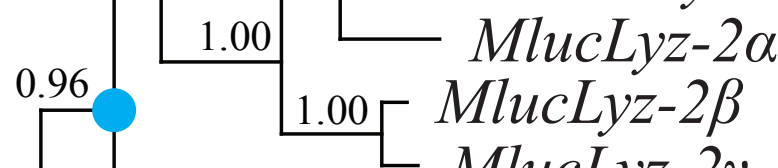

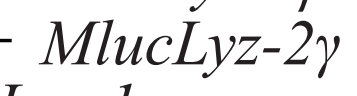

$0.28 \stackrel{1.00}{2}[$ MadvLyz-1

0.99 MricLyz-1

MsilLyz-1

1.00 -SkuhLyz-1

0.09

SkuhLyz-2

0.26 SornLyz-1

1.00 - IioLyz-1

0.50 SioLyz-2

0.40

¿.99 MleuLyz-1

0.99— MleuLyz-2

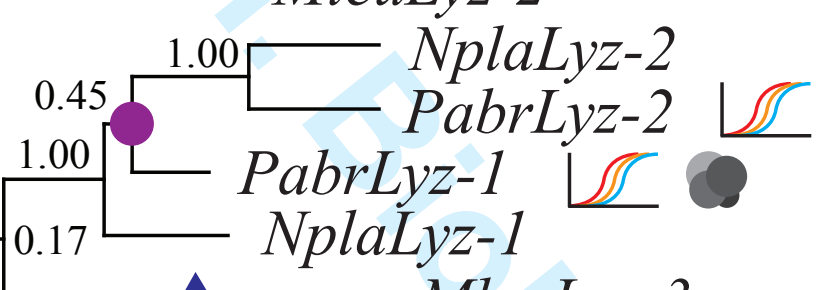

B. MleuLyz-3

B. beijingensis

M. fuliginosus

T. plicata

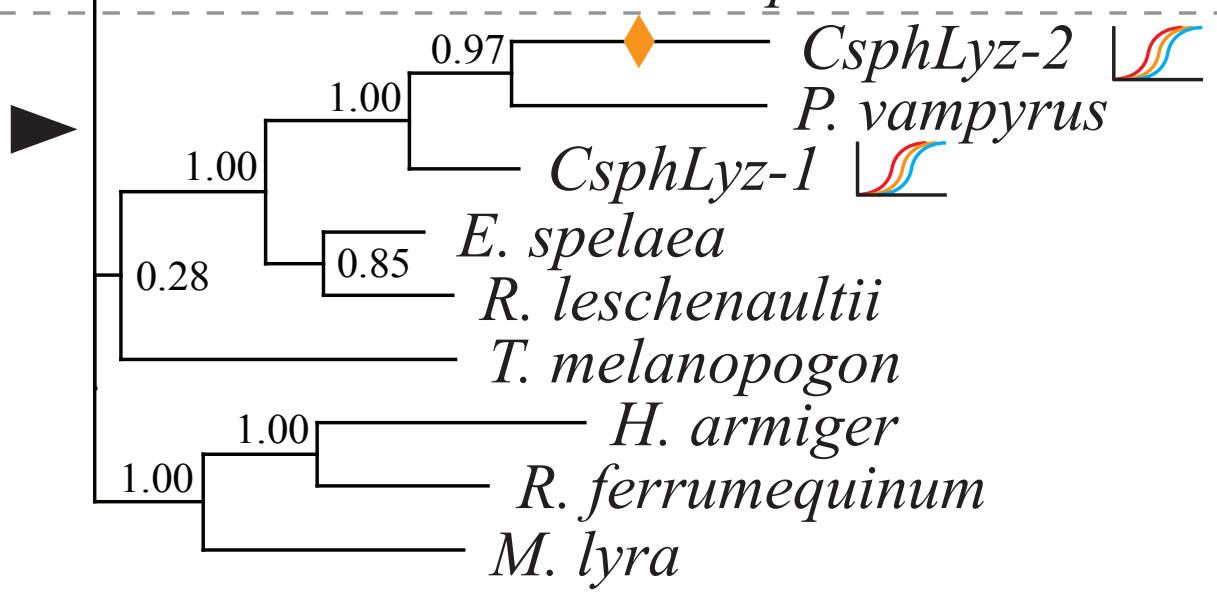

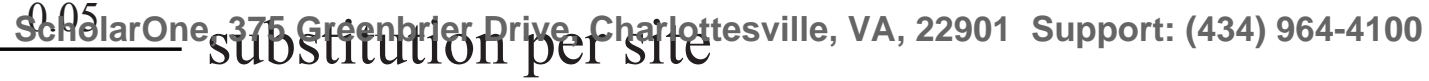



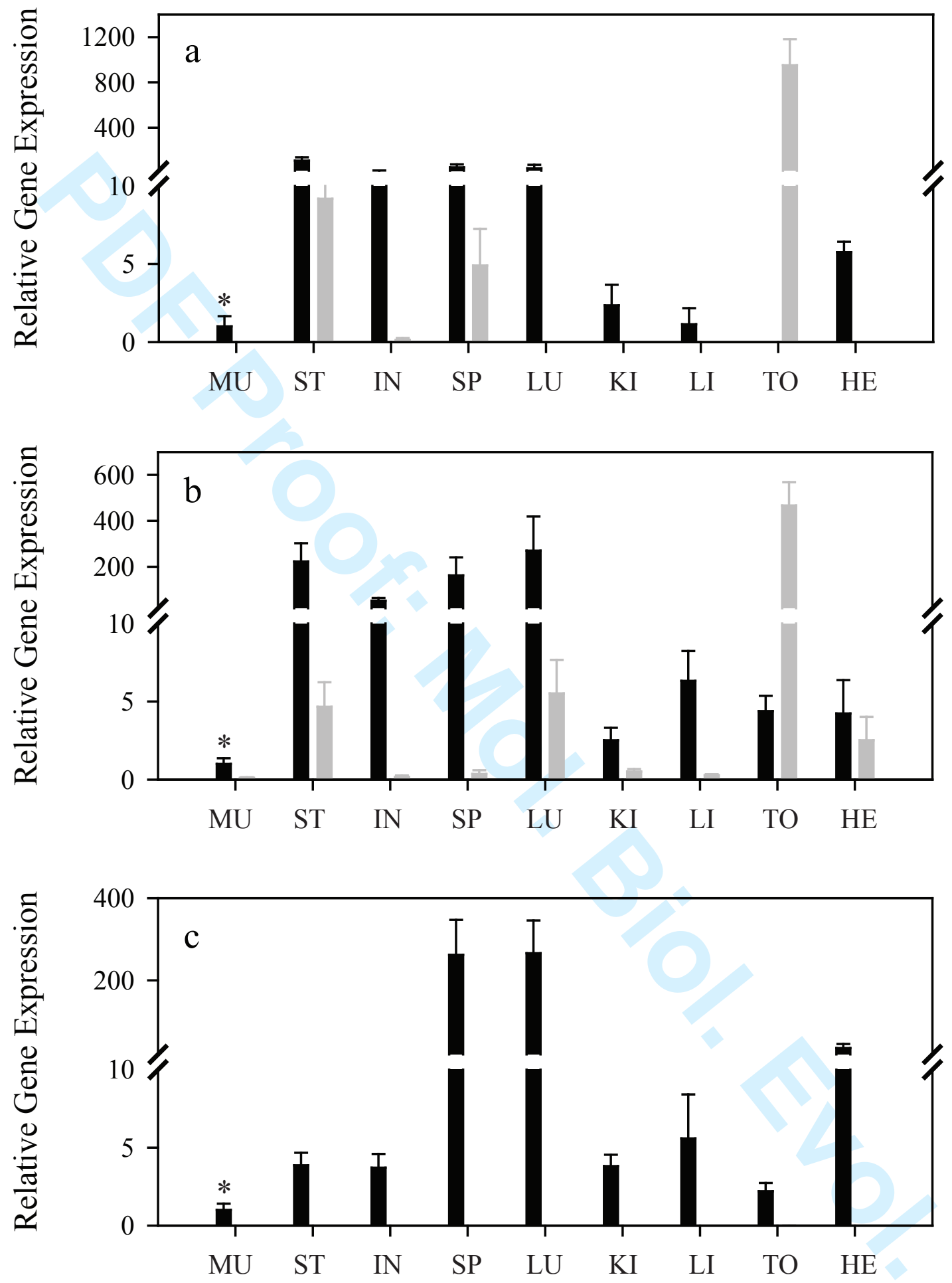

Organ 

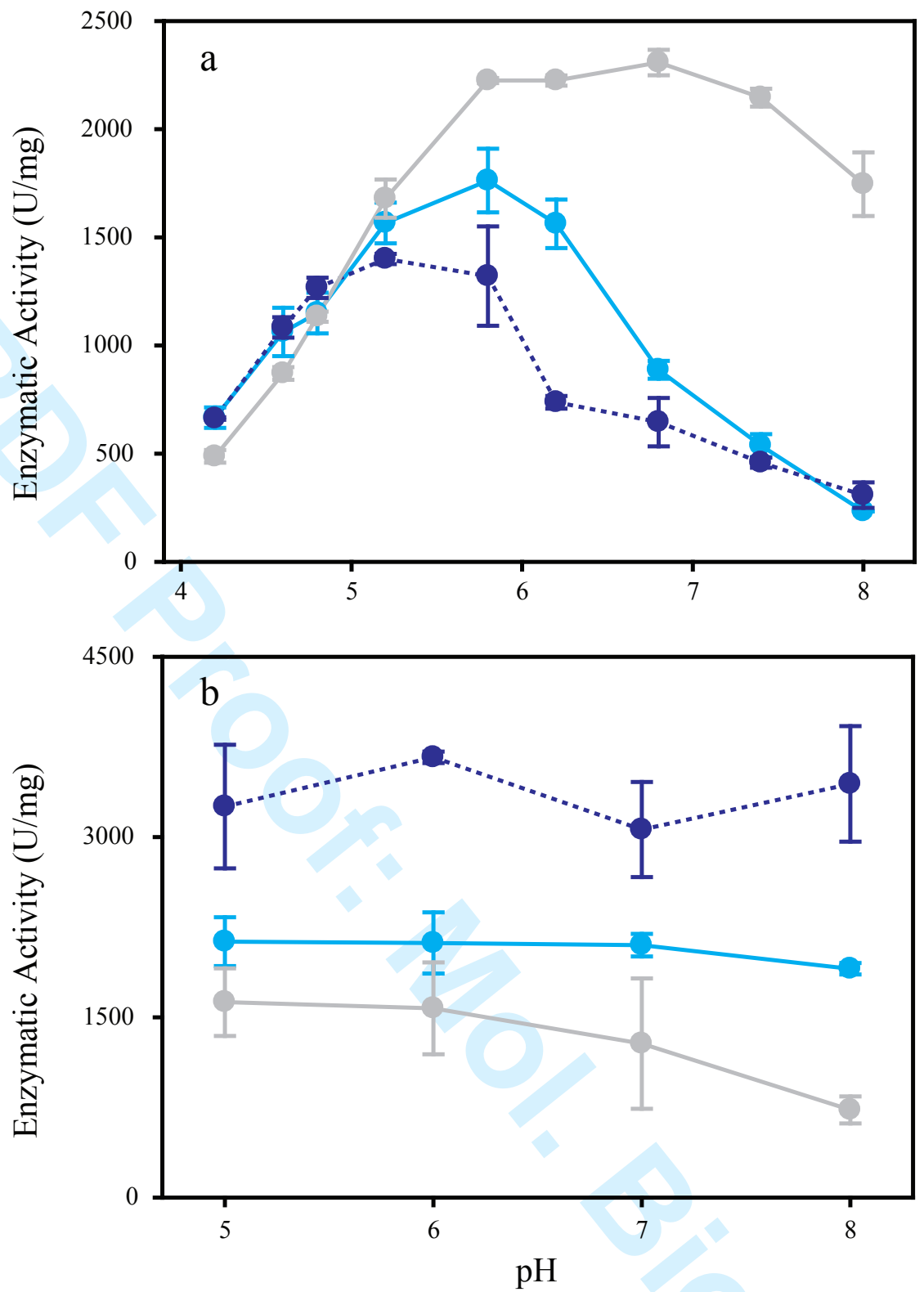